\title{
Granulomatous Mastitis with Erythema Nodosum: Case Report and Systematic Review
}

\author{
Nwanneoma Ngonadi, MSc ${ }^{1}$, Brian Florenzo, BS, BA ${ }^{1}$, Seth Martin, MD², R. Hal Flowers, MD² \\ 1 University of Virginia School of Medicine, Charlottesville, VA \\ 2 Department of Dermatology, University of Virginia, Charlottesville, VA
}

\section{ABSTRACT}

Although once considered a rare association, GM associated with EN is increasingly recognized, and since 1987, a total of 28 reports have detailed 59 cases (including the present case). Here, we provide a comprehensive review of all 58 reported cases of this association, including ours. We found a preponderance of cases occurred in females of Turkish ancestry around the fourth decade of life in the peripartum period. Arthralgia is the most common additional symptom. The majority of cases responded to treatment with oral corticosteroids, but some required the addition of other immunosuppressants and/or definitive surgical excision.

\section{INTRODUCTION}

First described in 1972 by Kessler and Wolloch, granulomatous mastitis (GM) is a rare idiopathic chronic inflammatory disease in women of childbearing age, often mimicking breast carcinoma or breast abscess ${ }^{1,2}$. It often presents in women with a recent history of pregnancy or lactation ${ }^{2}$ as a unilateral mass in any quadrant of the breast except the subareolar area, frequently with skin or lymph node involvement ${ }^{1}$. The etiology of GM remains unknown, and it is often a diagnosis of exclusion with non-specific features on histopathologic evaluation 1. Therapeutic management depends on severity of disease, and a tailored approach may include close observation and follow up, immunosuppressive medications, and/or surgical management. While prior reviews of GM have been performed, the association of GM with erythema nodosum (EN) has not been well-characterized. We present a classic case of EN in a patient with GM. A comprehensive review of the literature revealed numerous other similar cases, which we have compiled here in order to describe common features of this association.

\section{CASE}

A 34-year-old Hispanic female patient, gravida 5 para 3 , with history of diabetes mellitus and hypothyroidism initially presented with progressively worsening right sided breast pain in the setting of an enlarging ipsilateral breast mass. She denied any recent history of breastfeeding or nipple discharge and was over two years postpartum. She was given amoxicillinclavulanate twice daily for ten days for presumed mastitis without improvement. Mammogram showed an irregular and indistinct high-density mass in the upper outer quadrant of the right breast with associated abnormal lymph nodes; March 2022 Volume 6 Issue 2 
subsequent core needle biopsy of the breast mass diagnosed granulomatous mastitis. Approximately three weeks from the onset of her breast symptoms, she developed progressively enlarging painful nodules on her knees, lower extremities, and forearms.

Physical examination in the dermatology clinic revealed a $5 \times 8 \mathrm{~cm}$ tender indurated plaque on right lateral breast with no erythema, discharge or fluctuance (Figure 1A). Exam also showed multiple tender, erythematous subcutaneous nodules scattered over the extensor surfaces of bilateral upper and lower extremities (Figure 1B). Punch biopsy of a characteristic nodule on the right forearm exhibited a mixed septal and lobular panniculitis with acute neutrophilic inflammation with granulomatous changes, consistent with erythema nodosum. Chest X-ray, HIV testing and QuantiFERON Gold testing were performed and were normal/negative. The patient was initiated on a prednisone taper starting at $0.5 \mathrm{mg} / \mathrm{kg} /$ day. She experienced initial improvement

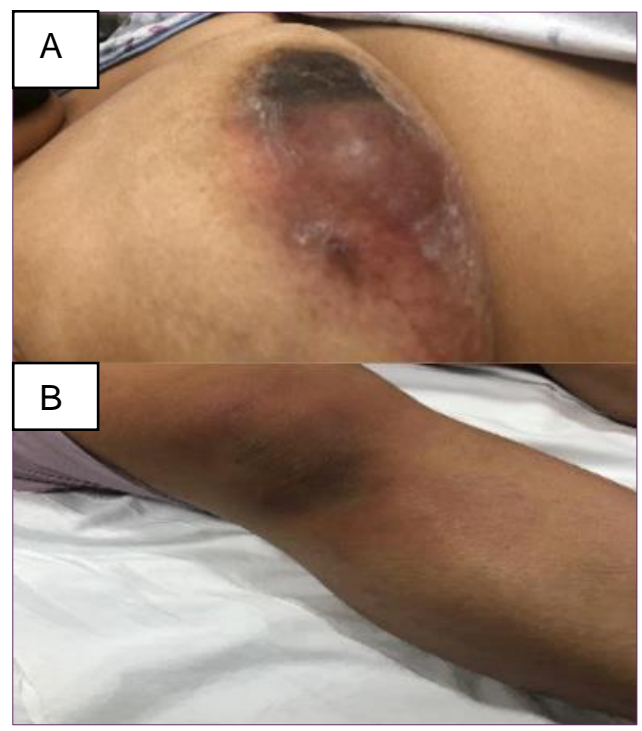

Figure 1. A) Granulomatous Mastitis B)

Erythema Nodosum

on prednisone but was transitioned to methotrexate after a flare. The size and induration of the breast mass steadily decreased on this therapy with eventual resolution of the erythema nodosum over the next three months.

\section{METHODS}

\section{Protocol and Research Strategy}

This systemic literature review was completed in accordance with the Preferred Reporting Items for Systemic Reviews and Meta-Analysis (PRISM) criteria. Using these guidelines, we entered (granulomatous mastitis) AND (erythema nodosum) in two databases (PubMed and Ovid MEDLINE) up to April 2021, from which 51 records were identified.

\section{Study Selection and Measures}

The 51 records were screened and examined. Those without a full copy of the manuscript in English, non-human studies, and studies with a well-established etiology other than granulomatous mastitis were excluded. The remaining records were imported into Zotero, and duplicates were removed. In the end, the 27 studies with a total of 58 patient reports that met our criteria were analyzed in addition to our case. Case data retrieved included demographic information, gestational status/history, disease duration, treatment efficacy, accompanying symptoms, as well as pathology and laboratory results

\section{RESULTS}

Demographic Characteristics of Patients with Concurrent GM/ EN

Current literature provided cases of GM/EN where parturition status, age, and ethnic

March 2022 Volume 6 Issue 2

(c) 2022 THE AUTHORS. Published by the National Society for Cutaneous Medicine. 
data are included, the results of which are summarized in Table 1. Overall, the average age of onset for $\mathrm{GM} / \mathrm{EN}$ was $32.9 \pm 4.91$ years (with a range 23-43 years old). Of these cases, 29 (49.2\%) patients were postpartum when their symptoms first developed. Some patients presented as early as 2-3 months post-partum while others developed GM/EN years later, though this data was limited by inconsistent reporting. Among the reports that included specific timeframes, eight (13.6\%) patients were pregnant when their symptoms first developed, six $(10.2 \%)$ were nulliparous, and sixteen (27.1\%) patients had an unknown gestational status.

With respect to demographics, GM/ EN has been reported in every racial group. However, a predominance of cases was reported in women of Turkish ancestry (64.4\%), with Hispanic patients $(10.1 \%)$ at a distant second 3-15. Additional affected groups can be found in Table 1, and include European, East Asian, and Persian individuals amongst others.

\section{Histopathology and Laboratory Findings}

Thirty-two described GM/EN cases in the literature include relevant breast biopsy data, details of which can be seen in Table 2. Of these, 32 (100\%) showed granulomatous infiltrate, $15 \quad(46.9 \%)$ contained lymphocytic infiltrate, $14(43.8 \%)$ noted the presence of neutrophils, $9(28.1 \%)$ had ductal/lobular inflammation or fibrosis, 6 (18.8\%) contained abscesses, 2 (6.3\%) stained for gram positive bacilli, and 1 $(3.1 \%)$ contained acid-fast bacilli. Descriptive histological reports of EN lesions associated with IGM were rarely reported, and the two available showed a granulomatous infiltrate with histiocytes, lymphocytes, multinucleate giant cells, and rare eosinophils $(10,15)$.
Patients with GM/EN showed several notable serologic abnormalities, which are exhibited in Table $3 . \quad$ Significant abnormalities included 11 cases with leukocytosis (range of $11.1-19.3 \mathrm{k} / \mu \mathrm{L}$ ), 14 cases with elevated erythrocyte sedimentation rate (range $34-114 \mathrm{~mm} / \mathrm{hr}$.), and 12 cases with increased C-reactive protein (range 15-374,000 mg/L). Pertinent negative findings in some cases included normal liver function, creatinine, BUN, calcium, angiotensin-converting enzyme, prolactin levels, and no evidence of autoantibodies $3,4,6,16,17$.

\section{Disease Course and Treatment Efficacy}

Overall, the average duration of GM was $14 \pm 9.9$ weeks, with many patients experiencing symptoms for more than 20 weeks $8,11,18$. The mean time for $E N$ to appear was $1.60 \pm 2.43$ weeks after the initial presentation of the $\mathrm{GM}$, with a range of $0-8$ weeks. The most common additional symptom reported was arthralgias, which was present in 22 cases. The arthralgias experienced by these patients tended to be polyarticular and common joints affected included knees, wrists, and shoulders. Additional symptoms or findings included fever in 12 cases, dyspnea or cough in 2 patients, and the presence of general malaise and multinodular goiter in 1 case each.

Of the $59 \mathrm{GM} / \mathrm{EN}$ patients, 15 (25.4\%) had failed antibiotic regimens before being worked up for inflammatory syndromes, and 29 patients $(49.2 \%)$ were successfully treated with an initial course oral corticosteroid. Generally, cases of recurrent or unresponsive GM/EN were successfully treated with more aggressive measures such as surgery, higher steroid doses, or additional drug combinations. There were two reported cases of patients being 
switched to higher doses of oral corticosteroids after failing other treatment regimens, with one patient requiring up to 60 $\mathrm{mg} /$ day of prednisone ${ }^{19}$.

Table 1. Demographic Factors in Patients with Co-occurring EN and GM

\begin{tabular}{|lr|}
\hline Trait & Cases of GM and EN \\
\hline Age; average ( $\boldsymbol{n})$ & $32.9+/-4.91(57)$ \\
\hline Gestational Status; $\boldsymbol{n}$ (\%) & $29(49.2)$ \\
Pregnant-Partum & $8(13.6)$ \\
Nulliparous & $6(10.2)$ \\
Unknown & $16(27.1)$ \\
Ethnicity; $\boldsymbol{n}$ (\%) & $38(64.4)$ \\
Turkish & $6(10.1)$ \\
Hispanic & $5(8.5)$ \\
European & $4(6.8)$ \\
East Asian & $3(5.1)$ \\
Persian & $1(1.7)$ \\
South Asian & $1(1.7)$ \\
African American & $1(1.7)$ \\
Unknown & \\
\hline
\end{tabular}

Table 2. Histological Profile of GM and EN Lesions in Literature

\begin{tabular}{|lr|}
\hline Pathology Finding & $\begin{array}{l}\text { Frequency of Described Pathology Findings, } \\
\mathbf{n}(\%)\end{array}$ \\
\hline Granulomatous Infiltrate & $32(100 \%)$ \\
\hline Lymphocytes & $15(46.9 \%)$ \\
Neutrophils & $14(43.8 \%)$ \\
Ductal/ Lobular Inflammation or Fibrosis & $9(28.1 \%)$ \\
Abscesses & $6(18.8 \%)$ \\
Other Bacteria & $2(6.3 \%)$ \\
Acid Fast Bacilli & $1(3.1 \%)$ \\
\hline
\end{tabular}


Table 3. Compiled Additional Findings in GM with EN Cases as Reported in Literature

\begin{tabular}{|lc|}
\hline Finding & Number of Reported Cases \\
\hline Additional Symptom $(\boldsymbol{n})$ & 22 \\
Arthritis & 12 \\
Fever & 2 \\
Myspnea/ Cough & 1 \\
Goiter & 1 \\
Abnormally Elevated Lab Values (range) & $14(34-114 \mathrm{~mm} / \mathrm{hr})$ \\
ESR & $12(15-374,000 \mathrm{mg} / \mathrm{L})$ \\
CRP & $11(11.1-19.3 \mathrm{k} / \mathrm{uL})$ \\
\hline WBC & \\
\hline
\end{tabular}

Though 31 cases $(52.5 \%$ of all GM/EN patients) responded to oral corticosteroids, 5 cases did not adequately respond and required excisional surgery of the GM or mastectomy ${ }^{6,11}$. In addition, five cases of GM/EN recurred after successful treatment with steroids, of which 2 eventually underwent excisional surgery ${ }^{6}$, 1 was switched to a higher steroid dose ${ }^{19}$, and 2 were switched to a higher steroid dose plus methotrexate ${ }^{21,26}$.

Three cases treated with corticosteroids failed to completely resolve the GM and patients were left with a residual mass ${ }^{20,1}$. Overall, corticosteroids resolved symptoms in $69.0 \%$ of patients that took them as a first line therapy. Recurrences occurred in $11.9 \%$ of patients, $7.1 \%$ were left with a residual mass, and $11.9 \%$ did not respond to corticosteroid therapy. The mean time for EN to resolve after the initiation of oral glucocorticoids was $3.3 \pm 4.7$ weeks 4,6 , 18. The response of GM to treatment was more delayed, as most patients experienced a gradual reduction in the size of breast masses until resolution or treatment failure $1,4,9,20$.

Eleven patients $(18.6 \%)$ in our study were successfully treated with surgical excision, mastectomy, or drainage, and there were no cases in which surgical treatment failed to resolve symptoms. Additional treatments mentioned in the literature are summarized in Table 4. Patients generally responded well to combination therapy with corticosteroids plus excisional surgery or another immunosuppressant. At least one patient was treated successfully with NSAIDs alone ${ }^{16}$, while two others resolved with anti-TB therapy 8 and doxycycline, respectively ${ }^{15}$.

\section{DISCUSSION}

To our knowledge, this is the largest systematic review examining the growing association, successful treatments, and disease course of granulomatous mastitis with erythema nodosum. Our review again demonstrated a predominance of cases in 
Table 4A. Overview of Recorded Treatments for GM with EN

\begin{tabular}{|c|c|c|c|c|}
\hline Treatment (N) & $\begin{array}{l}\text { Patients with Complete } \\
\text { Resolution (N) }\end{array}$ & $\begin{array}{l}\text { Recurrence after Initial } \\
\text { Resolution (N) }\end{array}$ & $\begin{array}{l}\text { Residual Mass Remaining After } \\
\text { Treatment (N) }\end{array}$ & $\begin{array}{l}\text { No resolution } \\
\text { (N) }\end{array}$ \\
\hline Corticosteroids (43) & 31 & 5 & 3 & 5 \\
\hline $\begin{array}{l}\text { Surgical Excision, Drainage, or } \\
\text { Mastectomy (11) }\end{array}$ & 11 & & & \\
\hline $\begin{array}{l}\text { Corticosteroids + Surgical Excision } \\
\text { (3) }\end{array}$ & 2 & 1 & & \\
\hline Corticosteroids + Methotrexate (4) & 3 & & 1 & \\
\hline Corticosteroids + Azathioprine (1) & 1 & & & \\
\hline Colchicine (1) & 1 & & & \\
\hline Anti-Tuberculosis Regimen (1) & 1 & & & \\
\hline NSAIDs (1) & 1 & & & \\
\hline Antibiotics & 1 & & & \\
\hline Refused Treatment (1) & & & & 1 \\
\hline
\end{tabular}

Table 4B. Treatment Substitution for Recurrent or Unresponsive GM with EN

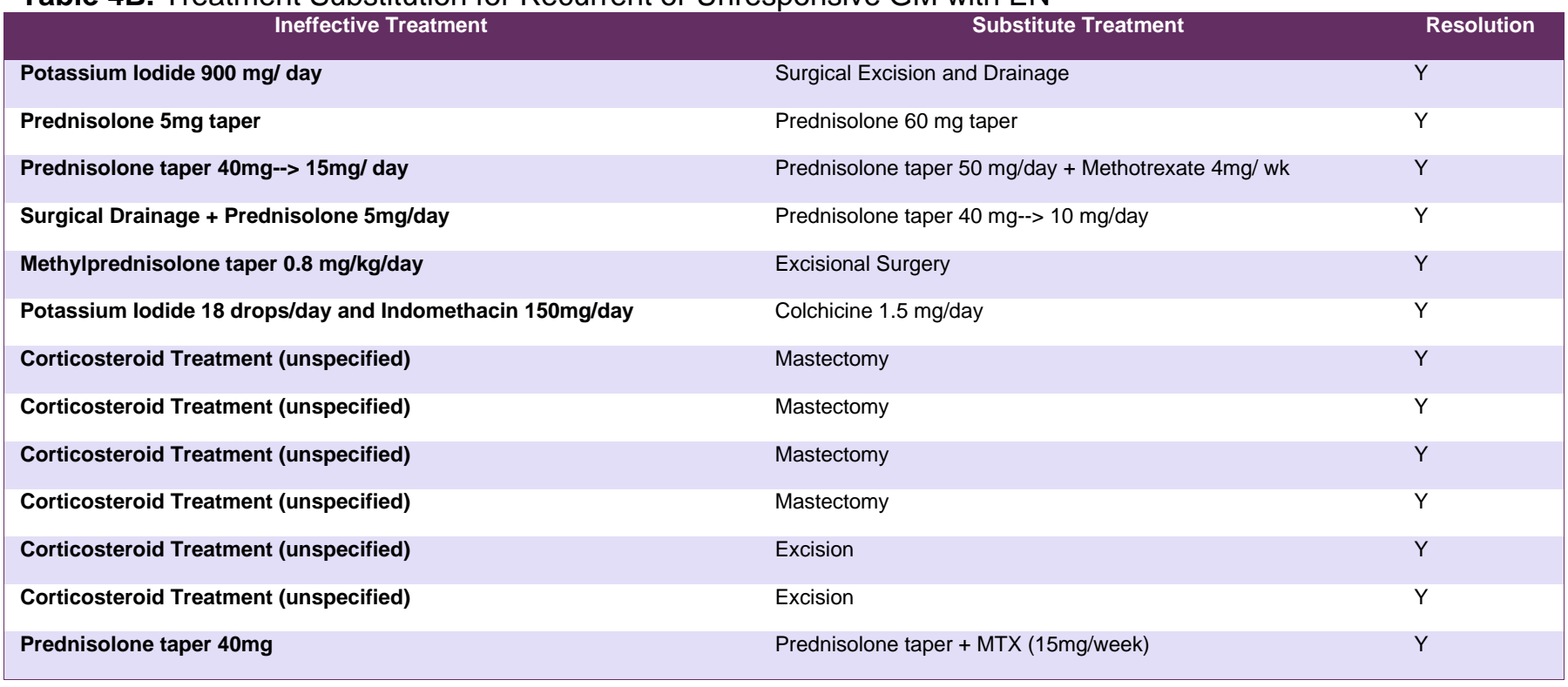

parous females in their late 20s and early 30s. Two previous reviews of 29 and 17 GM/EN cases found the average age of onset to be 32.9 and 31.4 respectively, similar to average age of 32.9 in this review 12, 21. Many previous reports have noted a possible association between parturition status and IGM. To our knowledge, no prior GM/EN reviews have examined post-partum status and very few reports specified the exact timing of prior births, which is why precisely correlating the two has remained elusive.

Analysis of two prior cohorts consisting of 11 and $6 \mathrm{GM} / \mathrm{EN}$ patients respectively revealed that $63.6 \%$ and $66.7 \%$ of patients had a history of parturition, which are higher than our finding of $49.2 \% 4,3$. Given that this is the first systemic review to examine this metric, this difference may be the result of a 
higher volume of patients or the fact that a significant portion of reports $(27.1 \%)$ failed to include parturition history. One prior review specifically examined the occurrence of $\mathrm{GM} / \mathrm{EN}$ in actively pregnant patients. They found a similar concurrence $(17.6 \%)$ to our finding of $13.6 \%{ }^{12}$. In addition to pregnancy, multiple sources reported an increased incidence of GM in women with oral contraceptive use, alpha-1 antitrypsin deficiency, and/or hyperprolactinemia 1, 3, 22, 7. The relationship between these factors and the development of the disorder remains an area of potential further study.

While the exact etiology of GM remains unknown, several literature sources have suggested that autoimmune processes are critical to disease pathogenesis and progression. Recent work by Koksal et al. in 2019 and $2020^{23,} 24$ revealed correlations between specific HLA types and increased levels of the proinflammatory cytokines IL-8 and IL-17. A similar study by Saydam et al. found abnormal levels of IL-22 and IL-23, but normal levels of IL-17 in patients with $\mathrm{GM}^{25}$. Correlating the frequency of specific HLA types and cytokine profiles with populations at higher risk for developing GM and/or EN is an area that requires further investigation and could potentially explain the increased incidence of $\mathrm{GM} / \mathrm{EN}$ in individuals of Turkish and Hispanic descent.

Regarding triggering events, some have proposed that it could be an immune response to ductal epithelial damage resulting in luminal secretions infiltrating the lobular connective tissue ${ }^{26}$. This could lead to a local inflammatory reaction that would prompt macrophage and lymphocyte migration to the region ${ }^{3,6}$. If true, this would also explain increased incidence of $\mathrm{GM}$ in patients who are hyperprolactinemic, currently pregnant, or recently pregnant since these conditions are known to increase luminal secretions. While only $28.1 \%$ of histology reports included in this review specifically mentioned ductal or lobular infiltrate, it is worth noting that its exclusion from descriptions does not necessarily indicate its absence, and its role in GM/EN should continue to be studied. While the common temporal relationship between GM and pregnancy, lactation, and post-partum status may suggest hormonal dysfunction as a driver, one must also take into account the normal fluctuation in cytokine profiles that occurs during and following pregnancy ${ }^{3,27}$. It is the opinion of the authors that the growing body of literature suggests a primary role of immune dysfunction in the pathogenesis of $\mathrm{GM} / \mathrm{EN}$.

The pathophysiologic relationship between GM and EN continues to be debated, but the frequency of co-occurrence has been welldescribed. All available GM histology reports in our study noted the presence of granulomatous infiltrate and nearly half $(46.9 \%)$ mentioned the presence of lymphocytes. Very similar histology was demonstrated in the few cases of EN that were biopsied. The histologic similarity is an association that previous reviews have pointed out ${ }^{12}$. Given these similarities, the two could be the result of the same underlying aberrant immune response triggered by changing cytokine levels that result in the deposition of immune complexes in the venules of the subcutaneous fat septae ${ }^{3}$.

As noted above, EN generally appeared 1-2 weeks after the initial IGM presentation. This correlates with the observation that autoimmune diseases and extramammary inflammatory responses, primarily nonspecific arthralgias and fever, are typically found in patients with a more severe GM presentation ${ }^{1,3,6}$. The frequent 
observation of arthritis in patients with GM/EN even prompted Parperis et al. ${ }^{12}$ to recently propose the eponym "GMENA" to describe this disease constellation of symptoms. Such associations potentially indicate that these symptoms arise from a more pronounced manifestation of the same immunologic dysfunction that leads to IGM alone.

There remains no consensus on the treatment of GM associated with EN. Because GM commonly mimics numerous infectious etiologies, patients (25.4\%) were often initially started on antibiotic treatments, including anti-tuberculosis regimens, vancomycin, and various beta-lactams, nearly all of which were ineffective $16,11,13,18$, 19. Based on this review, oral prednisone at a minimum of $40 \mathrm{mg} /$ day appears to be a reliable and efficacious first line therapy and can be useful in sparing patients more invasive treatments. 29 (69\%) GM/EN patients who were given oral steroids experienced complete amelioration of their symptoms without recurrences or the need for additional intervention. For those who were resistant to such treatment, increasing the corticosteroid dose, adding a diseasemodifying antirheumatic drug, and surgical excision were all shown to be effective strategies in resolving symptoms. None of the patients treated with these strategies were unresponsive, and it is worth noting that not a single patient treated with surgical excision or mastectomy experienced any recurrences or residual masses. Given the invasive, potentially disfiguring nature of this option, we would recommend reserving this as a secondary approach in accordance with patient preferences. An additional option would be to add a second immunosuppressive medication to an existing corticosteroid regimen, but the risk/ benefit ratio of such treatments in relation to excisional surgery for treating this condition remains unclear.

\section{CONCLUSION}

There is a growing body of evidence that GM is associated with extra-mamillary symptoms such as EN, polyarthralgia, and fever in a minority of cases. The mechanism of the disease remains largely unknown, although aberrant cytokine levels, increased luminal secretions, hormonal dysregulation, and other drivers of immune dysfunction are implicated in its pathogenesis.

The compilation of literature sources, including our present case, is highly suggestive that EN can occur as a reactive process secondary to or in conjunction with GM. Thus, female patients of childbearing age who present with EN should also be evaluated for GM and vice versa. Because extramammary symptoms are correlated with more severe cases of GM, it is crucial for physicians, especially dermatologists, to be aware of the association between EN and IGM in order to avoid delays in treatment and spare the patient from more invasive interventions.

\section{Conflict of Interest Disclosures: None}

Funding: None

Corresponding Author:

R. Hal Flowers IV, MD

Department of Dermatology

University of Virginia

1215 Lee Street,

Charlottesville, VA 22908

Email: RF9RJ@hscmail.mcc.virginia.edu

\section{References:}

1. Noma, M., Masahiro, O., Matsuura, K., \& Itamoto, T. Granulomatous Mastitis with Erythema Nodosum That Responded to Low-Dose Steroid: Case Report and Literature Review of Nine Patients. Case Reports in 
Clinical Medicine. 2014;3: 402-406. doi:

10.4236/crcm.2014.37089.

2. Halim NA, Uthman I, Rammal R, Assi HI. Idiopathic Granulomatous Mastitis Presenting as a Breast Pseudotumor: Case Reports with Review of the Literature. Soy M, ed. Case Rep Rheumatol. 2018;2018:4264012. doi:10.1155/2018/4264012

3. Şener Bahçe Z, Aktaş H. Patients with idiopathic granulomatous mastitis accompanied by erythema nodosum. Int $J$ Clin Pract. Published online December 10, 2020:e13928. doi:10.1111/ijcp.13928

4. Akın M, Karabacak H, Esendağlı G, et al. Coexistence of idiopathic granulomatous mastitis and erythema nodosum: successful treatment with corticosteroids. Turk J Med Sci. 2017;47(5):1590-1592. doi:10.3906/sag-1611-100

5. Atak T, Sagiroglu J, Eren T, Ali Özemir I, Alimoglu O. Strategies to treat idiopathic granulomatous mastitis: retrospective analysis of 40 patients. Breast Dis. 2015;35(1):19-24. doi:10.3233/BD-140373

6. Çetin K, Sıkar HE, Güllüoğlu BM. Idiopathic granulomatous mastitis with erythema nodosum: Is it a variant of clinical presentation indicating treatment resistance? A retrospective cohort study. Breast J. 2020;26(9):1645-1651. doi:10.1111/tbj.13944

7. Vural S, Ertop P, Ceyhan K, Şanli H. An Unusual Cause of Oligoarthritis and Erythema Nodosum: Idiopathic Granulomatous Mastitis. Arch Rheumatol. 2017;32(1):71-75. doi:10.5606/ArchRheumatol.2017.5952

8. Bes C, Soy M, Vardi S, Sengul N, Yilmaz F. Erythema nodosum associated with granulomatous mastitis: report of two cases. Rheumatol Int. 2010;30(11):15231525. doi:10.1007/s00296-009-1109-y

9. Gümüş $M$, Akkurt $Z M$, Gümüş $H$. Is erythema nodosum coexisting with lesions of the breast a suggestive sign for idiopathic granulomatous mastitis? Turk J Surg. 2018;34(1):71-73. doi:10.5152/turkjsurg.2017.3161

10. Polat M, Kaya H. Plantar erythema nodosum associated with granulomatous mastitis. Indian $\mathrm{J}$ Dermatol Venereol Leprol. 2016;82(2):202-204. doi:10.4103/0378-6323.164219

11. Kalaycı TÖ, Koruyucu MB, Apaydın M, Etit D, Varer M. Idiopathic Granulomatous Mastitis Associated with Erythema Nodosum. Balk Med J. 2016;33(2):228-231. doi:10.5152/balkanmedj.2015.150089

12. Parperis K, Achilleos S, Costi E, Vardas M. Granulomatous mastitis, erythema nodosum and arthritis syndrome: case-based review. Rheumatol Int. 2021;41(6). doi:10.1007/s00296-021-04820-8

13. Lucas R, Gussman D, Polis RL, Rattigan MI, Matulewicz TJ. Idiopathic granulomatous mastitis with erythema nodosum simulating breast abscess in pregnancy: A case report. Obstet Med. 2014;7(1):3739. doi:10.1177/1753495X13502474
14. Zabetian S, Friedman BJ, McHargue C. A case of idiopathic granulomatous mastitis associated with erythema nodosum, arthritis, and reactive cough. JAAD Case Rep. 2016;2(2):125-127. doi:10.1016/j.jdcr.2016.01.011

15. Fruchter R, Castilla C, Ng E, Pomeranz MK, Femia AN. Erythema nodosum in association with idiopathic granulomatous mastitis: a case series and review of the literature. J Eur Acad Dermatol Venereol. 2017;31(9):e391-e393. doi:10.1111/jdv.14194

16. Adams DH, Hubscher SG, Scott DG. Granulomatous mastitis--a rare cause of erythema nodosum. Postgrad Med J. 1987;63(741):581-582. doi:10.1136/pgmj.63.741.581

17. Weber J, Gros D, Blaison G, Martin T, Storck D, Pasquali J. Mastite granulomateuse, érythème noueux et oligoarthrite. À propos d'une observation. Rev Médecine Interne. 1994;15(3):190-192. doi:10.1016/S0248-8663(05)82147-2

18. Olfatbakhsh A, Beheshtian T, Djavid GE. Granulomatous Mastitis, Erythema Nodosum, and Oligoarthritis in a Pregnant Woman. Breast $J$. 2008;14(6):588-590. doi:10.1111/j.15244741.2008.00653.x

19. Goldberg J, Baute L, Storey L, Park P. Granulomatous mastitis in pregnancy. Obstet Gynecol. 2000;96(5, Part 2):813-815. doi:10.1016/S0029-7844(00)01051-6

20. Alungal J, Abdulla MC, Narayan R. Idiopathic granulomatous mastitis with erythema nodosum and polyarthritis. Reumatismo. 2016;68(2):97-99. doi:10.4081/reumatismo.2016.844

21. Choi EC, Wong SBJ, Ho S-AJ. Idiopathic Granulomatous Mastitis and Erythema nodosum - A Unifying Pathophysiology? Australas J Dermatol. 2021;62(1):e149-e153. doi:10.1111/ajd.13463468138.12604

22. Binesh F, Shiryazdi M, Bagher Owlia M, Azimi S. Idiopathic granulomatous mastitis, erythema nodosum and bilateral ankle arthritis in an Iranian woman. BMJ Case Rep. 2013;2013. doi:10.1136/bcr-2012-007636

23. Koksal H. Human leukocyte antigens class I and II in patients with idiopathic granulomatous mastitis. $A m \mathrm{~J}$ Surg. 2019;218(3):605-608. doi:10.1016/j.amjsurg.2019.01.038

24. Koksal H, Vatansev H, Artac H, Kadoglou N. The clinical value of interleukins-8, -10 , and -17 in idiopathic granulomatous mastitis. Clin Rheumatol. 2020;39(5):1671-1677. doi:10.1007/s10067-020-049258

25. Saydam M, Yilmaz KB, Sahin M, et al. New Findings on Autoimmune Etiology of Idiopathic Granulomatous Mastitis: Serum IL-17, IL-22 and IL-23 Levels of Patients. J Invest Surg. Published online February 11, 2020:1-5. doi:10.1080/08941939.2020.1725190

26. Nakamura T, Yoshioka K, Miyashita T, et al. Granulomatous mastitis complicated by arthralgia and March 2022 Volume 6 Issue 2 
erythema nodosum successfully treated with

prednisolone and methotrexate. Intern Med Tokyo Jpn.

2012;51(20):2957-2960.

doi:10.2169/internalmedicine.51.7846

27. Kruse N, Greif M, Moriabadi NF, Marx L, Toyka KV,

Rieckmann P. Variations in cytokine mRNA expression during normal human pregnancy. Clin Exp Immunol. 2000;119(2):317-322. doi:10.1046/j.13652249.2000.01123.x

28. Hida T, Minami M, Kawaguchi H, Oshiro Y, Kubo Y. Case of erythema nodosum associated with granulomatous mastitis probably due to Corynebacterium infection. J Dermatol. 2014;41(9):821-823. doi:10.1111/13

29. Al-Khaffaf BH, Shanks JH, Bundred N. Erythema Nodosum - An Extramammary Manifestation of Granulomatous Mastitis. Breast J. 2006;12(6):569-570. doi:10.1111/j.1524-4741.2006.00348.x

30. Salesi M, Karimifar M, Salimi F, Mahzouni P. A case of granulomatous mastitis with erythema nodosum and arthritis. Rheumatol Int. 2011;31(8):1093-1095. doi:10.1007/s00296-009-1273-0

31. Jacquin-Porretaz C, Devalland C, Delapparent T, Nardin C, Dupond A-S. [Idiopathic granulomatous mastitis associated with erythema nodosum]. Ann Dermatol Venereol. 2019;146(8-9):571-576.

doi:10.1016/j.annder.2019.04.023

32. Donn W, Rebbeck P, Wilson C, Gilks CB. Idiopathic granulomatous mastitis. A report of three cases and review of the literature. Arch Pathol Lab Med. 1994;118(8):822-825. 